PROCEEDINGS OF THE

AMERICAN MATHEMATICAL SOCIETY

Volume 94, Number 3, July 1985

\title{
TRANSLATION PROPERTIES OF SETS OF POSITIVE UPPER DENSITY
}

\author{
VITALY BERGELSON AND BENJAMIN WEISS ${ }^{1}$
}

\begin{abstract}
Generalizing a result of Raimi we show that there exists a set $E \subset \mathbf{N}$ such that if $A \subset \mathbf{N}$ is a set with positive upper density, then there exists a number $k \in \mathbf{N}$ such that $d^{*}((A+k) \cap E)>0$ and $d^{*}\left((A+k) \cap E^{c}\right)>0$. Some extensions and further results are also obtained.
\end{abstract}

The purpose of this note is to generalize the following theorem due to Raimi (see [1]).

THEOREM. There exists a set $E \subset \mathbf{N}$ such that, whenever $r \in \mathbf{N}$ and $\mathbf{N}=\bigcup_{1 \leqslant i \leqslant r} D_{i}$ there exists $1 \leqslant i \leqslant r$ and $k \in \mathbf{N}$ with

$$
\left|\left(D_{i}+k\right) \cap E\right|=\omega \text { and }\left|\left(D_{i}+k\right) \cap E^{c}\right|=\omega .
$$

Raimi's proof used a topological result about N. Another proof was given by Ryll-Nardzewski [2]. See also [3].

Raimi's theorem is topological in nature and it is natural to ask whether a density version holds.

The upper density $d^{*}(A)$ of a set $A \subset \mathbf{N}$ is defined by

$$
d^{*}(A) \stackrel{\text { def }}{=} \limsup _{n \rightarrow \infty}|A \cap[1, n]| / n,
$$

where $[1, n]=\{1, \ldots, n\}$. If the limit exists and is positive, then we say that $A$ has positive density $d(A)>0$. If $\mathbf{N}=\bigcup_{1 \leqslant i \leqslant r} D_{i}$ then at least one of the sets $D_{i}$ has positive upper density. Thus the following theorem is clearly a strengthening of Raimi's result.

THEOREM 1. There exists a set $E \subset \mathbf{N}$ such that for any $A \subset \mathbf{N}$ with $0<d^{*}(A)$ there exists a $k \in \mathbf{N}$ such that

$$
d^{*}((A+k) \cap E)>0 \text { and } d^{*}\left((A+k) \cap E^{c}\right)>0 \text {. }
$$

Received by the editors April 26, 1984.

1980 Mathematics Subject Classification. Primary 10L10; Secondary 10K25.

Key words and phrases. Density, normal number.

${ }^{1}$ The research of the second author was supported in part by U.S.-Israel Grant N 1927/79.

(c) 1985 American Mathematical Society $0002-9939 / 85 \$ 1.00+\$ .25$ per page 
In fact, the assertion of Theorem 1 holds for every normal set $E \subset \mathbf{N}$ (see definition below). Theorem 1 is actually a corollary of the following

TheOREM $1^{\prime}$. If $E \subset \mathbf{N}$ is normal and $A \subset \mathbf{N}$ has positive upper density $d^{*}(A)$ then

$$
d^{*}((A+k) \cap E)>0 \text { and } d^{*}\left((A+k) \cap E^{c}\right)>0
$$

holds for all $k \in \mathbf{Z}$ with at most $\left[-\log _{2} d^{*}(A)\right]$ exceptions.

An even stronger result holds, namely

THEOREM 2. If $E \subset \mathbf{N}$ is normal, $A \subset \mathbf{N}$ has upper density $d^{*}(A)$ and $\varepsilon>0$, then

$$
d^{*}((A+k) \cap E)>\frac{1}{2} d^{*}(A)-\varepsilon \text { and } d^{*}\left((A+k) \cap E^{c}\right)>\frac{1}{2} d^{*}(A)-\varepsilon
$$

holds for all $k \in \mathbf{Z}$ with at most $\left[d^{*}(A) / 4 \varepsilon^{2}\right]$ exceptions.

Before presenting the proofs of Theorems 1 and 2, we give the definition and some basic properties of normal sets.

To any set $A \subset \mathbf{N}$ we attach the $(0,1)$-sequence $a_{n}=1_{A}(n)$ which is its indicator function.

Definition. Let $\left\{a_{n}\right\}_{n=1}^{\infty}$ be a $(0,1)$-sequence. Let $B_{k}=b_{1} b_{2} \cdots b_{k}, k \geqslant 1$, be any $(0,1)$-word of length $k$. Denote by $D\left(B_{k}, m\right)$ the number of occurances of the block $B_{k}$ as a sub-block in the block $a_{1} a_{2} \cdots a_{m}$, i.e.

$$
D\left(B_{k}, m\right)=\mid\left\{n \in\{1, \ldots, m-k+1\}: a_{n+j-1}=b_{j} \text { for } 1 \leqslant j \leqslant k\right\} \mid,
$$

the sequence $\left\{a_{n}\right\}_{n=1}^{\infty}$ is normal if $\lim _{m \rightarrow \infty} D\left(B_{k}, m\right) / m=2^{-k}$ for all $k \geqslant 1$ and all $B_{k}$.

A set $A \subset \mathbf{N}$ is normal if $1_{A}(n)$ is a normal sequence.

It is, perhaps, not obvious that such sets do exist, but actually almost every $(0,1)$-sequence is normal (if one views $(0,1)$-sequences as dyadic expansions of numbers in $[0,1]$ with usual Lebesgue measure). There are also numerous explicit constructions of normal sequences (see [4-6]).

For example $11011100101110 \cdots$ is a normal sequence (this sequence is formed by the sequence $1,2,3, \ldots$ written in base 2 ).

If $E$ is a normal set, then obviously $d(E)=d\left(E^{c}\right)=\frac{1}{2}$.

If $E$ is a normal set, then $d(E \cap(E+k))=\frac{1}{4}$ for all $k \in \mathbf{Z} \backslash\{0\}$. To see this, note that $1_{E \cap(E+k)}(n)=1$ iff $n \in E$ and $n-k \in E$. Each $(0,1)$-word $i_{1} i_{2} \cdots i_{k+1}$ of length $k+1$ appears in $E$ with frequency $1 / 2^{k+1}$ and, in exactly $2^{k-1}$ of these words, $i_{1}=i_{k+1}=1$. So, the frequency of those $n$ that satisfy $n \in E$ and $n-k \in E$ is equal to $2^{k-1} / 2^{k+1}=\frac{1}{4}$.

In the same fashion one shows that if $E$ is normal set, then

$$
d\left(E \cap\left(E+k_{1}\right) \cap\left(E+k_{2}\right) \cap \cdots \cap\left(E+k_{m}\right)\right)=2^{-(m+1)}
$$

for any integers $0<k_{1}<k_{2}<\cdots<k_{m}$. 
It is not difficult to see that the same holds if we replace some of the sets $E+k_{i}$ by $E^{c}+k_{i}$. So we have the following

LEMMA 1. Let E be normal set and let

$$
E^{\alpha}= \begin{cases}E & \text { if } \alpha=1, \\ E^{c} & \text { if } \alpha=-1 .\end{cases}
$$

Then for any distinct integers $k_{1}, k_{2}, \ldots, k_{m}$ and any $(-1,1)$-word $\alpha_{1} \alpha_{2} \cdots \alpha_{m}$,

$$
d\left(\bigcap_{i=1}^{m}\left(E^{\alpha_{i}}+k_{i}\right)\right)=2^{-m} .
$$

Proof of Theorem $1^{\prime}$. Let $k_{1}, \ldots, k_{m}$ be distinct integers for which $(*)$ fails. That is, for each $1 \leqslant i \leqslant m$ there is an $\alpha_{i} \in\{-1,1\}$ such that $d^{*}\left(\left(A+k_{i}\right) \cap E^{\alpha_{i}}\right)=0$. Shifting both $A+k_{i}$ and $E^{\alpha_{i}} k_{i}$ units to the left, we obtain

$$
d^{*}\left(A \cap\left(E^{\alpha_{i}}-k_{i}\right)\right)=0, \quad i=1,2, \ldots, m,
$$

and therefore

$$
d^{*}\left(A \cap \bigcup_{i=1}^{m}\left(E^{\alpha_{i}}-k_{i}\right)\right)=0
$$

This, in turn, implies

$$
\begin{aligned}
d^{*}(A) & =d^{*}\left(A \cap\left(\mathbf{N} \backslash \bigcup_{i=1}^{m}\left(E^{\alpha_{i}}-k_{i}\right)\right)\right) \\
& =d^{*}\left(A \cap \bigcap_{i=1}^{m}\left(E^{-\alpha_{i}}-k_{i}\right)\right) \\
& \leqslant d^{*}\left(\bigcap_{i=1}^{m}\left(E^{-\alpha_{i}}-k_{i}\right)\right)=2^{-m}
\end{aligned}
$$

(see Lemma 1), and therefore $-\log _{2} d^{*}(A) \geqslant m$.

LEMMA 2. Let $(X, B, \lambda)$ be a probability space, and let $\mathscr{E}$ be a (finite or infinite) collection of measurable subsets of $X$, such that, for some $\delta \geqslant 0,\left|\lambda(E)-\frac{1}{2}\right| \leqslant \delta$ for all $E \in \mathscr{E}$ and $\left|\lambda(E \cap F)-\frac{1}{4}\right| \leqslant \delta$ for any two distinct set $E, F \in \mathscr{E}$. If $A \subset X$ is measurable and $\varepsilon>\sqrt{2 \delta \lambda(A)}$, then the inequality

$$
\left|\lambda(A \cap E)-\frac{1}{2} \lambda(A)\right|<\varepsilon \quad\left(\text { or, equivalently }\left|\lambda\left(A \cap E^{c}\right)-\frac{1}{2} \lambda(A)\right|<\varepsilon\right)
$$

holds for $E \in \mathscr{E}$ with at most $\lambda(A) / 2\left(\varepsilon^{2}-2 \delta \lambda(A)\right)$ exceptions.

Proof of Lemma 2. First note that if $E, F \in \mathscr{E}, E \neq F$, then

$$
\begin{array}{rlrl}
\left|\lambda\left(E^{c}\right)-\frac{1}{2}\right| & \leqslant \delta, & & \left|\lambda\left(E \cap F^{c}\right)-\frac{1}{4}\right| \leqslant 2 \delta, \\
\left|\lambda\left(E^{c} \cap F\right)-\frac{1}{4}\right| & \leqslant 2 \delta, & \left|\lambda\left(E^{c} \cap F^{c}\right)-\frac{1}{4}\right| \leqslant 3 \delta,
\end{array}
$$

and therefore

$$
\left|\lambda(E \cap F)-\lambda\left(E^{c} \cap F\right)-\lambda\left(E \cap F^{c}\right)+\lambda\left(E^{c} \cap F^{c}\right)\right| \leqslant 8 \delta .
$$


Using characteristic functions, we can rewrite the last inequality as

$$
\begin{aligned}
\left|\int\left(21_{E}-1\right)\left(21_{F}-1\right) d \lambda\right| & =\left|\int\left(1_{E}-1_{E^{c}}\right)\left(1_{F}-1_{F^{c}}\right) d \lambda\right| \\
& =\left|\int\left(1_{E} 1_{F}-1_{E^{c}} 1_{F}-1_{E} 1_{F^{c}}+1_{E^{c}} 1_{F^{c}}\right) d \lambda\right| \\
& =\left|\int\left(1_{E \cap F}-1_{E^{c} \cap F}-1_{E \cap F^{c}}+1_{E^{c} \cap F^{c}}\right) d \lambda\right| \leqslant 8 \delta .
\end{aligned}
$$

Define

$$
\begin{gathered}
\mathscr{E}_{+}=\left\{E \in \mathscr{E}: \lambda(A \cap E) \geqslant \frac{1}{2} \lambda(A)+\varepsilon\right\}, \\
\mathscr{E}_{-}=\left\{E \in \mathscr{E}: \lambda(A \cap E) \leqslant \frac{1}{2} \lambda(A)-\varepsilon\right\} .
\end{gathered}
$$

Lemma 2 asserts that $\left|\mathscr{E}_{+} \cup \mathscr{E}_{-}\right| \leqslant \lambda(A) / 2\left(\varepsilon^{2}-2 \delta \lambda(A)\right)$. We shall actually show that

$$
\max \left(\left|\mathscr{E}_{+}\right|,\left|\mathscr{E}_{-}\right|\right) \leqslant \lambda(A) / 4\left(\varepsilon^{2}-2 \delta \lambda(A)\right)
$$

We shall carry out the calculations for $\mathscr{E}_{+}$only. Suppose $E_{1}, \ldots, E_{n}$ are distinct sets in $\mathscr{E}_{+}$. We denote by $1_{i}$ the characteristic function of $E_{i}, 1 \leqslant i \leqslant n$.

From the definition of $\mathscr{E}_{+}$we obtain

$$
\begin{aligned}
\varepsilon & \leqslant \frac{1}{n} \sum_{i=1}^{n} \lambda\left(A \cap E_{i}\right)-\frac{1}{2} \lambda(A) \\
& =\int\left(\frac{1}{n} \sum_{i=1}^{n} 1_{A} 1_{E_{i}}-\frac{1}{2} 1_{A}\right) d \lambda \\
& =\int 1_{A}\left(\frac{1}{n} \sum_{i=1}^{n} 1_{E_{i}}-\frac{1}{2}\right) d \lambda \\
& =\int 1_{A}\left(\frac{1}{2 n} \sum_{i=1}^{n}\left(21_{i}-1\right)\right) d \lambda .
\end{aligned}
$$

Applying the classical Cauchy-Schwarz inequality we can continue:

$$
\begin{aligned}
& \leqslant\left[\int 1_{A}^{2} d \lambda \cdot \int \frac{1}{4 n}\left(\sum_{i=1}^{n}\left(21_{i}-1\right)^{2}\right) d \lambda\right]^{1 / 2} \\
& =\left[\int 1_{A} d \lambda \cdot \frac{1}{4 n^{2}} \sum_{i=1}^{n} \sum_{j=1}^{n} \int\left(21_{i}-1\right)\left(21_{j}-1\right) d \lambda\right]^{1 / 2} \\
& =\left[\lambda(A) \cdot \frac{1}{4 n^{2}}\left(\sum_{i=1}^{n} \int\left(21_{i}-1\right)^{2} d \lambda+2 \sum_{1 \leqslant i<j \leqslant n} \int\left(21_{i}-1\right)\left(21_{j}-1\right) d \lambda\right)\right]^{1 / 2}
\end{aligned}
$$

Observing that $\left(21_{i}-1\right)^{2} \equiv 1$ and using inequality (1) we can continue:

$$
\leqslant\left[\lambda(A) \cdot \frac{1}{4 n^{2}}(n+n(n-1) 8 \delta)\right]^{1 / 2} \leqslant\left[\lambda(A) \cdot\left(\frac{1}{4 n}+2 \delta\right)\right]^{1 / 2} .
$$


It follows that $\varepsilon^{2} \leqslant \lambda(A)(1 / 4 n+2 \delta)$, and therefore after elementary calculations we obtain $n \leqslant \lambda(A) / 4\left(\varepsilon^{2}-2 \delta \lambda(A)\right)$.

The proof of the inequality for $\mathscr{E}_{-}$is essentially the same.

Proof of Theorem 2. Suppose $E \subset \mathbf{N}$ is a normal set and let $A \subset \mathbf{N}$ and $\varepsilon>0$ be given.

For a set $B \subset \mathbf{N}$ we shall write $d_{n}(B)=\frac{1}{n} \mid B \cap[1, n]$, so that $d^{*}(B)=$ $\lim \sup _{n \rightarrow \infty} d_{n}(B)$. Define

$$
\begin{aligned}
\mathscr{K} & =\left\{k \in \mathbf{Z}: d^{*}((A+k) \cap E)<\frac{1}{2} d^{*}(A)-\varepsilon\right\} \\
& =\left\{k \in \mathbf{Z}: d^{*}(A \cap(E-k))<\frac{1}{2} d^{*}(A)-\varepsilon\right\}, \\
\mathscr{K}^{\prime} & =\left\{k \in \mathbf{Z}: d^{*}\left((A+k) \cap E^{c}\right) \leqslant \frac{1}{2} d^{*}(A)-\varepsilon\right\} \\
& =\left\{k \in \mathbf{Z}: d^{*}\left(A \cap\left(E^{c}-k\right)\right) \leqslant \frac{1}{2} d^{*}(A)-\varepsilon\right\} .
\end{aligned}
$$

We shall prove Theorem 2 by showing that $\max \left(|\mathscr{K}|,\left|\mathscr{K}^{\prime}\right|\right) \leqslant d^{*}(A) / 4 \varepsilon^{2}$. Suppose that $|\mathscr{K}|>d^{*}(A) / 4 \varepsilon^{2}$. Let $k_{1}, k_{2}, \ldots, k_{n}$ be distinct numbers in $\mathscr{K}, n>$ $d^{*}(A) / 4 \varepsilon^{2}$. Choose a positive number $\delta$, such that $n>d^{*}(A) / 4\left(\varepsilon^{2}-2 \delta d^{*}(A)\right)$ and let $\left\{m_{i}\right\}_{i=1}^{\infty}$ be an increasing sequence of positive integers such that $d^{*}(A)=$ $\lim _{i \rightarrow \infty} d_{m_{i}}(A)$. Choose a number $i_{0}$ such that for all $i \geqslant i_{0}$ the following inequalitites hold:

$$
\begin{aligned}
& \left|d_{m_{i}}\left(\mathbf{N} \cap\left(E-k_{p}\right)\right)-\frac{1}{2}\right|<\delta \text { for all } 1 \leqslant p \leqslant n, \\
& \left|d_{m_{i}}\left(\mathbf{N} \cap\left(E-k_{p}\right) \cap\left(E-k_{q}\right)\right)-\frac{1}{4}\right|<\delta \text { for all } 1 \leqslant p<q \leqslant n, \\
& n>d_{m_{i}}(A) / 4\left(\varepsilon^{2}-2 \delta \cdot d_{m_{i}}(A)\right) .
\end{aligned}
$$

(The existence of a number $j_{0}$ is an immediate consequence of the normality of $E$.)

For $i \geqslant i_{0}$ and $1 \leqslant p \leqslant n$ put $A^{i}=A \cap\left[1, m_{i}\right] ; E_{p}^{i}=\left(E-k_{p}\right) \cap\left[1, m_{i}\right]$. Note that $d_{m_{i}}$ is a probability measure on the set of all subsets of $\left[1, m_{i}\right]$.

Inequalities (2) can be rewritten as

$$
\begin{aligned}
& \left|d_{m_{i}}\left(E_{p}^{i}\right)-\frac{1}{2}\right|<\delta \quad(1 \leqslant p \leqslant n), \\
& \left|d_{m_{i}}\left(E_{p}^{i} \cap E_{q}^{i}\right)-\frac{1}{4}\right|<\delta \quad(1 \leqslant p<q \leqslant n), \\
& n>d_{m_{i}}\left(A^{i}\right) / 4\left(\varepsilon^{2}-2 \delta \cdot d_{m_{i}}\left(A^{i}\right)\right) .
\end{aligned}
$$

By the proof of Lemma 2 there is at least one index $p_{i}, 1 \leqslant p_{i} \leqslant n$, such that

$$
d_{m_{i}}\left(A^{i} \cap E_{p_{i}}^{i}\right)>\frac{1}{2} d_{m_{i}}(A)-\varepsilon,
$$

Since $p_{i} \in[1, n]$ for all $i \geqslant i_{0}$, there is an infinite set $I$ of indices and a number $p$ such that $p_{i}=p$ for all $i \in I$.

Passing to the upper limit as $i \rightarrow \infty, i \in I$, we obtain from (3) $d^{*}(A \cap E-p)$ $\geqslant \frac{1}{2} d^{*}(A)-\varepsilon$ for some $p \in \mathscr{K}$ which contradicts the definition of $\mathscr{K}$. This shows that $|\mathscr{K}| \leqslant d^{*}(A) / 4 \varepsilon^{2}$. The proof that $\left|\mathscr{K}^{\prime}\right| \leqslant d^{*}(A) / 4 \varepsilon^{2}$ is essentially the same and is left to the reader.

It is natural to ask whether or not the results obtained here can be generalized to other groups. As a matter of fact even the group structure is irrelevant, and one can 
establish the following result: Let $\Phi$ denote some countably infinite family of one-to-one mappings $\varphi: \mathbf{N} \rightarrow \mathbf{N}$ (not necessarily onto), that acts freely on $\mathbf{N}$, i.e. for $\varphi \neq \varphi^{\prime}$ and all $n \in \mathbf{N}, \varphi(n) \neq \varphi^{\prime}(n)$. Then there exists a set $E$ such that for all sets $A$ with positive upper density, both $d^{*}\left(A \cap \varphi^{-1}(E)\right)>0$ and $d^{*}\left(A \cap \varphi^{-1}\left(E^{c}\right)\right)>0$ hold for $\varphi \in \Phi$ with at most $\left[-\log _{2} d^{*}(A)\right]$ exceptions. The proof goes along the lines of the proof of Theorem $1^{\prime}$, to be sure the notion of normality of $E$ is defined now with respect to $\Phi$. Because of the independence of the underlying random variables, the fact that $\Phi$ has no structure presents no obstacle and one easily establishes the existence of $\Phi$-normal sets $E$ that satisfy the property:

For every finite set $\Phi_{0} \subset \Phi$, and every choice of $a(\varphi) \in\{-1,1\}, \varphi \in \Phi_{0}$, we have

$$
\lim _{n \rightarrow \infty} \frac{1}{n}\left|\left\{i \leqslant n: \varphi(i) \in E^{a(\varphi)}, \varphi \in \Phi_{0}\right\}\right|=2^{-\left|\Phi_{0}\right|}
$$

where as usual $E^{1}=E$ and $E^{-1}=E^{c}$.

Then for $E$ one can take any $\Phi$ normal set. The details are straightforward and can safely be left to the reader.

Acknowledgement. The authors would like to thank Professor M. Perles of the Hebrew University for carefully reviewing an earlier version of this note. The present version owes a great deal to his comments both stylistic and mathematical.

\section{REFERENCES}

1. R. Raimi, Translation properties of finite partitions of the positive integers, Fund. Math. 61 (1968), 253-256.

2. C. Ryll-Nardzewski, Remark on Raimi's theorem on translations, Fund. Math. 61 (1968), 257-258.

3. N. Hindman, Ultrafilters and combinatorial number theory, Lecture Notes in Math., Vol. 751, Springer-Verlag, Berlin, 1979.

4. D. G. Champernowne, The construction of decimals normal in the scale of ten, J. London Math. Soc. 8 (1933), 254-260.

5. R. von Mises, Über Zahlenfolgen die ein Kollektiv-ahnliches Verhalten zeigen, Math. Ann. 108 (1933), 757-772.

6. H. Davenport and P. Erdös, Note on normal decimals, Canad. J. Math. 4 (1952), 58-63.

Institute of Mathematics and Computer Science, Hebrew University of Jerusalem, Givat RAM, 91904 JERUSALEM, ISRAEL

Current address (Vitaly Bergelson): Department of Mathematics, Ohio State University, Columbus, Ohio 43210 